\title{
Parametric studies on vibration characteristics of triply periodic minimum surface sandwich lattice structures
}

\author{
Ugur Simsek ${ }^{1,2} \cdot$ Tolga Arslan $^{2}$ • Baris Kavas ${ }^{1} \cdot$ Cemal Efe Gayir $^{1} \cdot$ Polat Sendur $^{2}$ (I)
}

Received: 24 August 2020 / Accepted: 22 September 2020 / Published online: 7 October 2020

(C) Springer-Verlag London Ltd., part of Springer Nature 2020

\begin{abstract}
Additive manufacturing has opened new avenues for the manufacturing of structures to achieve challenging engineering tasks. Gyroid, a unique example of such structures, exhibits many attractive properties, such as high stiffness-to-weight ratio and impact characteristics. This study aimed to evaluate the dynamic performance of gyroid structures made from HS188 using direct metal laser melting. The frequency response predictions of a finite element-based model of the gyroid sandwich structure were first validated against the modal testing in terms of its natural frequencies and mode shapes using the Dewesoft software. Subsequently, the effects of the plate and gyroid wall thickness on the dynamic characteristics of the structure were investigated by varying these across their expected limit ranges as part of a parametric study using the validated finite element model. The findings from the parametric study were validated against modal testing. Moreover, the performance of the aforementioned structure was compared with that of a solid structure with the same mass. The simulation results indicated that the dynamic characteristics of the gyroid structure can be improved considering the structure's frequency response by using parametric models. It was concluded that simulation and optimization tools will play a crucial role in additive manufacturing techniques to attain optimal mechanical properties of complex structures.
\end{abstract}

Keywords Additive manufacturing $\cdot$ Double gyroid $\cdot$ Frequency response $\cdot$ Modal testing $\cdot$ Design optimization

\section{Introduction}

A sandwich structure refers to two solid surfaces that are bonded by different density cores. These structures can be found in nature, such as bone tissue and wood [1]. Moreover, these structures are commonly used in the automotive, aerospace, aviation, and wind energy systems, owing to their competitive mechanical properties and lightweight characteristics. Two core types may exist, depending on the manufacturing methods: (i) a cellular structure, and (ii) a quasi-randomly distributed porous structure. In the former method, the core is fabricated by means of an interconnected network of surfaces or ligaments. The cellular core provides high resistance against bending and buckling forces, while maintaining a low

Polat Sendur

polat.sendur@ozyegin.edu.tr

1 Additive Manufacturing, GE Aviation, Kocaeli, Turkey

2 Mechanical Engineering Department, Ozyegin University, Cekmekoy, Istanbul, Turkey weight [2]. Its adjustable relative density, which may be as low as $2 \%$, enables the structure to dissipate into plastic deformation and heat under quasi-static and dynamic loading [3-7]. The low volume fraction of sandwich panels makes them ideal for isolation elements in heat transfer applications. Furthermore, the porous internal structure has an extended surface area, which significantly increases the convection inside the panels. These thermal capabilities of sandwich structures are currently being investigated and are viewed as strong candidates for applications in which isolation, cooling, or heating are important design considerations [7-9]. Owing to their superior properties of interest, sandwich structures are considered as indispensable in various engineering applications and fields, such as aviation, aerospace, automotive, transportation, packaging, personal protective equipment, and armor [10]. However, until the early 2000s, the true potential of sandwich structures was limited as a result of the difficulties associated with their manufacturing methods.

Various manufacturing methods are available for the production of metallic or polymeric core structures. Solid forming with welding, brazing, and investment casting is the most common manufacturing method for 
metals. Special types of cellular structures known as honeycombs have dominated the majority of applications owing to their ease of manufacturing. There have been many research studies on the mechanical properties of honeycomb structures in the literature [11]. Additive manufacturing (AM) technologies enable the production of highly complex structures that would otherwise be impossible or impractical to manufacture. With the aid of developing AM technologies, highly complex designs with complex features can be manufactured. Cellular structures are perfect examples of designs produced by AM technologies, as very complex unit cell types can be manufactured using this technology. Manufacturing and mechanical investigations into the lattice structure, which mimics the atomic distribution of metals, have been gaining increasing attention in recent years. Several types of FCC, BCC, FCCZ, and octet truss lattice structures have been studied in the literature [5, 12-15]. In addition to the mechanical analysis of proposed unit cell types, the applications of specific unit cells have been presented. Lattice structures have been optimized and manufactured with high precision in many structural applications in the literature [16-21]. However, despite their benefits, truss lattice structures exhibit several disadvantages, such as high stress concentration in the nodes, anisotropy, and inferior inspectability. Because of these challenges, research on the unit cell design for lattices has shifted toward the more recently implemented surface model family known as the triply periodic minimal surface (TPMS) [22].

The definitions of TPMS structures and preliminary demonstrations were initially published by the NASA scientist Alan H. Schoen [23] in 1970. TPMS surface models represent isotropic, continuous, periodic surfaces, elongating infinitely in three directions. The TPMS family primarily covers the Schwarz Primitive, Diamond, Shoen IWP, and Gyroid surface model types [24]. Afshar et al. [25] studied the mechanical characterization of Primitive and Diamond surfaces that were created using PolyJet technology. Abueidda et al. [26] investigated the mechanical properties of Primitive, IWP, and Neovius manufactured by selective laser sintering. With the aim of studying the microscopic characteristics of these structures, AlKetan et al. [27] fabricated strut, skeletal, and sheet-based cellular structures made of maraging steel using powder bed fusion, and observed the microscopic characteristics using a microscope. Gyroid structures with different porosity ratios have been tested to determine their torsional and compression characteristics [28]. Moreover, the specific mechanical performances of several TPMS lattice structures under various loading directions were examined and guidelines were defined for optimal lattice structure design [29]. Computational methods, particularly the finite element methods, have been used extensively for analyzing and characterizing the mechanical behavior of lattice structures. In one such study, an effective simulationbased approach for lattice design was proposed by deriving an empirical relation between the elastic moduli and volume fraction [30]. In another major study, the vibration behaviors of several TPMS-based geometries were analyzed computationally, and the effect of the wall thickness was investigated [31].

The double gyroid (DG), one of the well-known TPMS forms exhibiting robust mechanical properties, has been garnering interest in numerous practical applications in recent years. Maskery et al. [32] studied the energy absorption and failure modes of additively fabricated DG lattices, and highlighted the higher compressive strength-to-weight ratio of the DG. In another study, the mechanical properties of gyroid structures were examined by experimental testing and finite element analysis. The findings suggested that continuity of the gyroid surface results in lower stresses owing to the reduced stress concentrations throughout the structure [33].

In the aforementioned works, DG structures were mainly investigated in terms of their mechanical properties, such as the compressive stress-strain and energy absorption characteristics. However, in comparison, there are a limited number studies about the dynamic properties of these structures. One such study has shown that the vibration bandgaps may be adjusted by changing cell types and relative densities [34]. It obviously indicates that DG lattice structures can be utilized in vibration isolation and acoustic insulation applications. Moreover, most of these studies considered common TPMS lattices consisting of a certain number of unit cells made from a polymeric base material and were not experimentally validated. The proposed study focused on determining the dynamic characteristics of a novel structure composed of DG unit cells sandwiched between two sheet metals. The aim was to understand the effects of the main design parameters on the dynamic responses of the aforementioned structure. Such knowledge is important for designing more effective structures in real-world engineering applications. To this end, a finite element-based investigation was conducted to determine the modal and frequency response characteristics of the unique structures with different relative densities. Furthermore, the numerical studies were validated by performing modal testing. A design sensitivity study considering important design parameters was conducted as part of a parametric study.

The remainder of this paper is organized as follows: Firstly, the finite element modeling of the gyroid structures is explained in Section 2. The experimental setup for validating the models using the Dewesoft software is described in Section 3. Thereafter, a parametric study to understand the effects of the design parameters on the 
dynamic performance of gyroid structures is presented in Section 4. The verification of the parametric study by means of modal testing is outlined in Section 5. Finally, conclusions are presented in Section 6.

\section{Finite element model}

\subsection{Geometry of DG structures}

The DG model used in this study was modeled using the second method described in the work of Monkova et al. [35]. The mathematical approximation formula for the gyroid is presented in Eq. 1:

$$
\begin{aligned}
{\left[\cos \left(\frac{2 \pi}{\alpha} x\right) \cdot \sin \left(\frac{2 \pi}{\alpha} y\right)\right.} & +\cos \left(\frac{2 \pi}{\alpha} y\right) \cdot \sin \left(\frac{2 \pi}{\alpha} z\right) \\
& \left.+\cos \left(\frac{2 \pi}{\alpha} z\right) \cdot \sin \left(\frac{2 \pi}{\alpha} x\right)\right]^{2}=t^{2}
\end{aligned}
$$

where $\alpha$ is the unit cell size and $t$ controls the volume fraction. The surface model was generated by setting the parameter $t$ to 0 in MATLAB. The resulting surface was then exported to the stereolithography format (.stl) as a triangulated facet body. Smoothening of the surface was performed in the Unigraphics (UG) 12 software. Finally, two surfaces were generated using the "offset surface" command in two opposite directions, by setting the offset distance to half of the desired thickness. A solid block was created in the exact desired rectangular dimensions, and the block was trimmed by using both offset surfaces. The resulting geometry represented the solid body of the DG structure. The modeling steps implemented in UG software are illustrated in Fig. 1. The solid body was triangulated again and sliced to be sent to Concept Laser M2 for manufacturing.

Representative cubic specimens were manufactured to assess the quality of the manufacturing process. For this purpose, destructive metallographic and nondestructive metrological examinations were performed on the specimens. The void defects were observed in the downfacing areas of the specimen, as indicated by the cut-up section in Fig. 2. In powder bed AM modalities, powder is coated as a thin layer, and the laser selectively scans

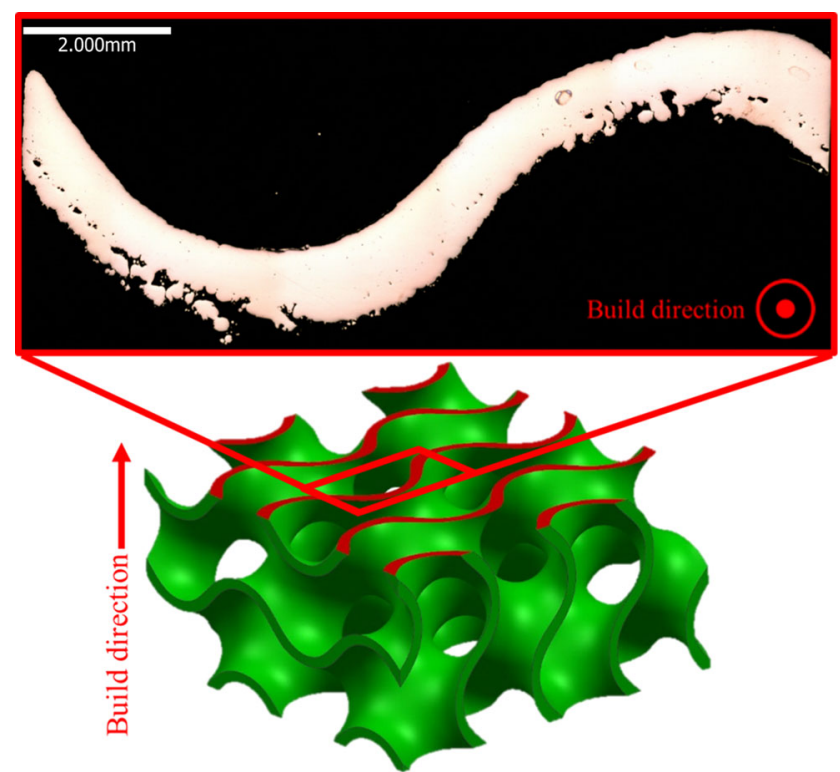

Fig. 2 Cut-up metallographic section of surface region parallel to build direction

the surface as dictated by the slice file. When the angle relative to the build direction is higher than $45^{\circ}$, heat is dissipated from the melt pool, mainly toward the powder underneath, compared to the previously solidified areas. This increases the likelihood of porosity and roughness on the down-facing surfaces. Although geometrical errors and build defects existed in the specimens, repetitively performed compressive tests demonstrated that the effects of these errors and defects were not detrimental to the mechanical properties of the specimens. The exemption of the mechanical responses from these defects could be explained by the ductile nature of the alloy [36]. The surface quality was improved when the angle was increased. Owing to the geometric complexity of the DG structure, hangover areas were inevitable.

Similar defects in the hangover areas were observed in another cross-section, as illustrated in Fig. 3. The region with porosity in the hangover areas disappeared in the direction of the up-facing surfaces. The surface intersection area in this image was manufactured as required. Such
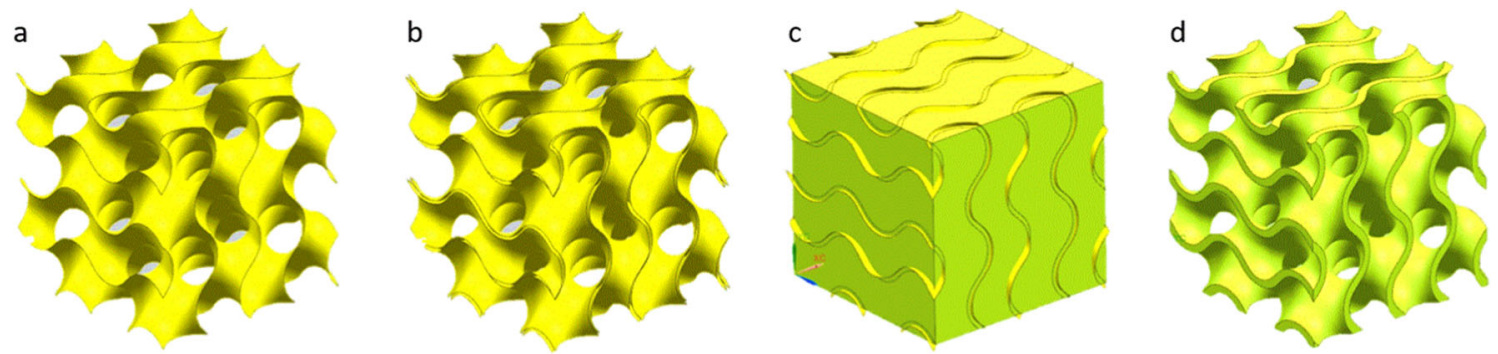

Fig. 1 DG structure geometry: a facet body, b trimmed surfaces, c $24 \times 24 \times 24 \mathrm{~mm}$ solid body, and d final $24 \times 24 \times 24 \mathrm{~mm}$ DG 


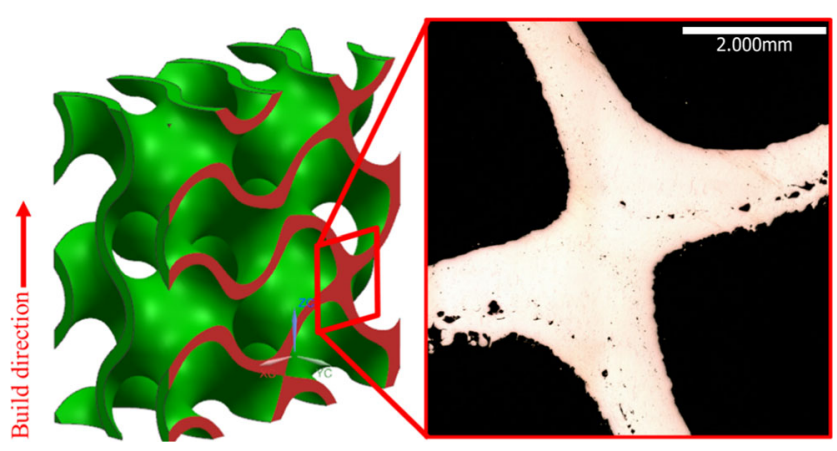

Fig. 3 Cut-up metallographic detail of intersection region perpendicular to build direction

intersections can be considered as important regions in TPMS structures, as the stress flow dissipates into the structure from these intersections.

Prior to the metallographic inspection, the side surface relative to the build direction of the specimens was scanned by an Alicona device with $a \times 5$ magnifying microscope lens. The resulting data were then exported in .stl format using the commercial software GOM Inspect. Owing to the model complexity, there was no datum to use as a reference; therefore, best fitting was performed as the most reliable alignment operation available. Because of the aforementioned expected building defects, most vertical surfaces were selectively used as best-fitting references in the geometry. Color-mapped images according to the distance between the CAD geometry and scanned data are presented in Fig. 4. The red shaded areas represent the overthe-surface areas, while the blue shaded areas represent the areas under the surface.

In the resulting image, it can be observed that significant profile drop-downs appeared in the hangover areas. These drop-downs consisted of small bumps and a very thin pvorous layer underneath, as mentioned previously. Relatively many vertical surfaces were colored in green by approximation to the perfect fit, while the visible up-facing regions were colored in blue. This means that, although the profile dropdown effects in the hangover areas were compensated for in the build direction, they still affected the up-facing surface profile.

In this study, a complete $192 \times 192 \times 25.6 \mathrm{~mm}$ DG structure was generated, consisting of $24 \times 24 \times 24 \mathrm{~mm}$ gyroid structures, each with 12 -mm unit cells. The complete gyroid structure was sandwiched between upper and lower sheets, each with a thickness of $0.8 \mathrm{~mm}$. The additively fabricated sandwich structure is illustrated in Fig. 5. The material of the sandwich gyroid structure is a widely used high-temperature alloy named as Haynes ${ }^{\circledR} 188$ (HS188). It is a commercially available super-alloy with excellent hightemperature corrosion resistance that enables it to be used in applications where temperature rises as high as $1095^{\circ} \mathrm{C}$ while maintaining its strength for prolonged exposures [37]. The main field of application of the alloy is gas turbines' hot sections. Its weldability characteristics make it a promising candidate to be used in additive manufacturing. However, there has been limited literature concerning an additively manufactured HS188 specimen. The material
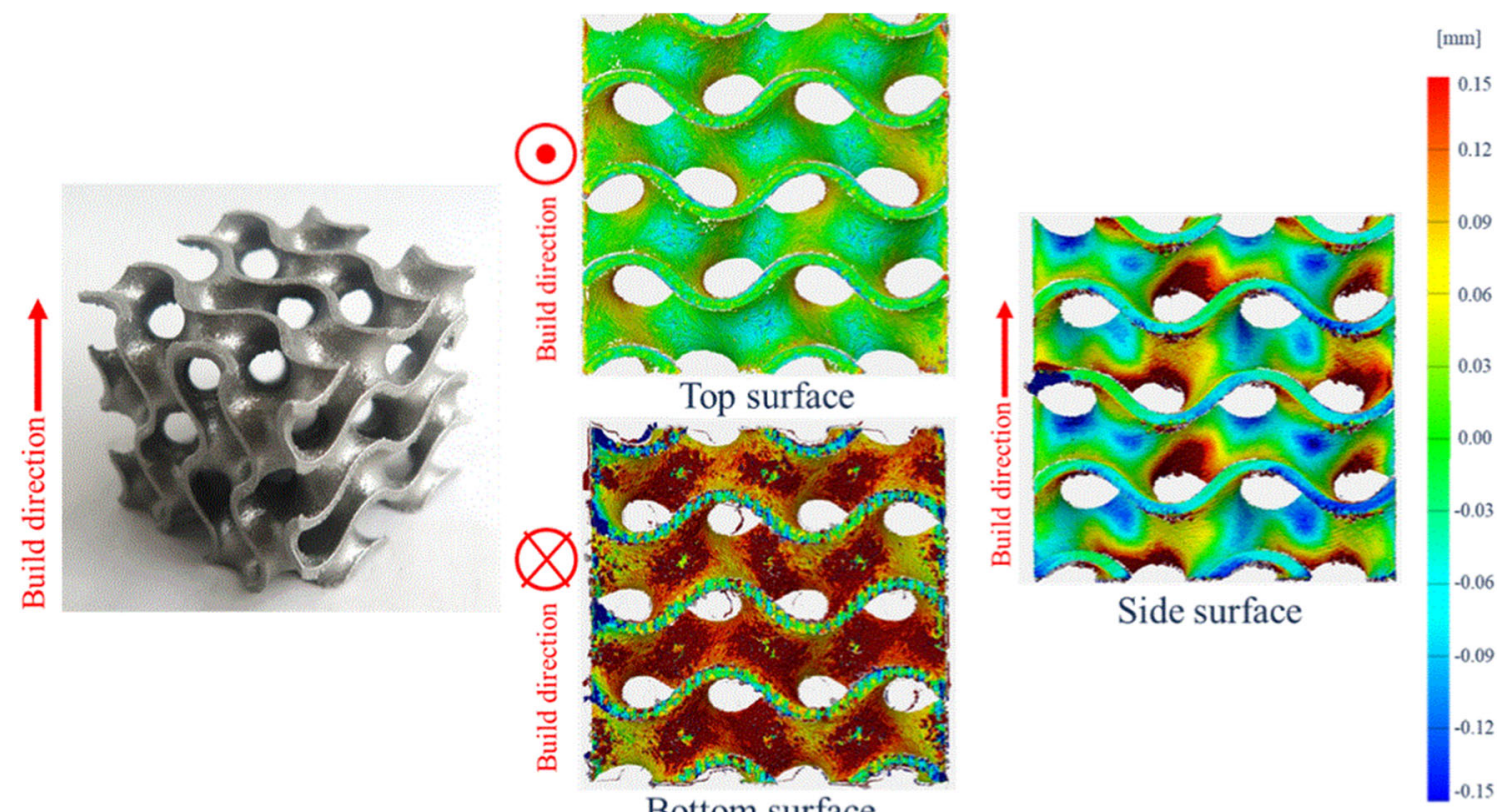

Fig. 4 Color mapped profile measurements of top, bottom, and side surfaces of 12-mm unit cell size DG structure 


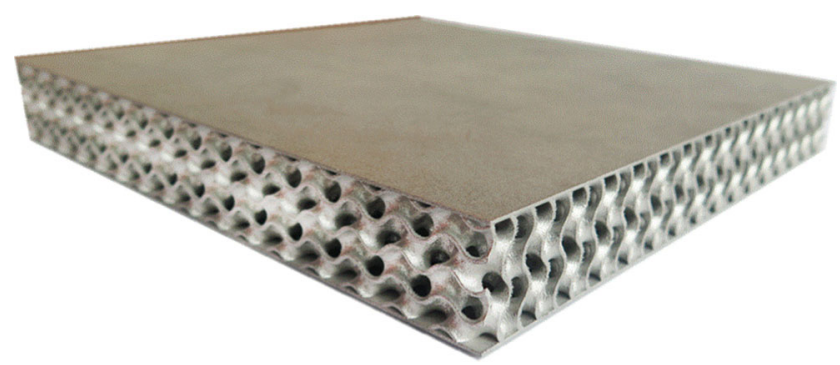

Fig. 5 Additively manufactured $192 \times 192 \times 25.6 \mathrm{~mm}$ sandwich gyroid structure

properties used in the finite element models are given in Table 1 [38].

\subsection{Finite element model}

Owing to the complex nature of the gyroid-based sandwich structures, it is challenging to create a finite element model (FEM) representing the CAD geometry accurately. The modeling techniques for such structures in the literature are quite limited. For example, Maskery et al. [22] explicitly modeled gyroid lattice structures with hexahedral meshes. In another study, 10-node tetrahedral elements were selected for the mesh owing to the geometric complexity of the gyroid structure [28]. Moreover, Simsek et al. [39] implemented a homogenization-based model by means of representative 3D hexagonal solid elements to improve the computational efficiency and accuracy. In the current work, the entire structure was modeled using shell elements. One alternative approach is 3D solid mesh modeling of the gyroid thicknesses. In this approach, the gyroid walls should be modeled by a couple of elements through the thickness to capture the stiffness of the structure correctly and avoid locking problems under bending loading. The use of multiple elements for the through-wall thickness results in computationally expensive solutions, thereby posing an obstacle for the considered parametric optimization study. However, the modeling of the gyroid wall with shell-type elements also exhibits certain disadvantages in terms of accuracy. The most significant shortcoming is that the surface thickness in the transition region of the gyroid structure cannot be modeled accurately. This is because the thickness is assigned symmetrically as the gyroid wall is mid-plane, and this approach leads to overlap zones which have a higher thickness value than the nominal value. In view of this explanation, it was expected that the shell meshing method would demonstrate notably superior computational performance compared with solid mesh modeling, and the accuracy of the shell mesh model for this unique structure was verified with the experimental results.

The FEM of the $192 \times 192 \times 25.6 \mathrm{~mm}$ gyroid structure was generated by combining the FEMs of the repetitive
Table 1 Material properties of HS188 [38]

\begin{tabular}{lll}
\hline Density $\left(\mathrm{g} / \mathrm{cm}^{3}\right)$ & Poison's ratio (-) & Young's modulus (GPa) \\
\hline 8.98 & 0.3 & 232 \\
\hline
\end{tabular}

patterns of the gyroid structure. This approach offered the advantage of obtaining a homogenous mesh model throughout the gyroid structure. Thereafter, finite element meshing was performed using the ANSA software. Both the plate and the gyroid structures were modeled with $2 \mathrm{D}$ finite elements. The average mesh size is determined as $0.6 \mathrm{~mm}$ by performing a mesh convergence study. There were 683,200 finite elements in the model, consisting of quadrilateral plate (CQUAD4) and triangular plate (CTRIA3) elements. The CTRIA3 elements constituted approximately $6 \%$ of the total element number. The plate and gyroid structure thicknesses were both $0.8 \mathrm{~mm}$. The total mass of the structure was $2.17 \mathrm{~kg}$, where the masses of the plates and gyroids were $1.63 \mathrm{~kg}$ and $0.54 \mathrm{~kg}$, respectively. The calculated overall mass of the computational model completely agreed with the fabricated test sample mass at $2.19 \mathrm{~kg}$. The FEM is illustrated in Fig. 6.

\subsection{Modal analysis and frequency response functions}

Modal analysis is generally applied to determine the dynamic characteristics of a mechanical structure. The frequency response functions (FRFs) and modal parameters of structures, such as the natural frequencies and mode shapes, can be determined by performing modal analysis of the FEM. The aforementioned gyroid sandwich structure is a multi-degree-of-freedom (MDOF) system. The motion equation of an MDOF mechanical system is presented in Eq. 2, described by the matrix equation, where $[M]$ is the

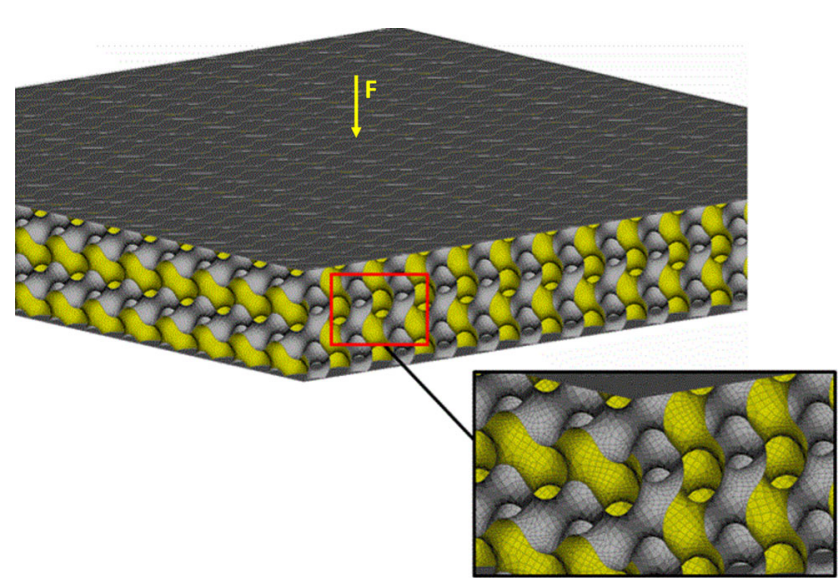

Fig. 6 FEM of the gyroid structure 
mass matrix, $[B]$ is the damping matrix, $[K]$ is the stiffness matrix, and $\mathrm{P}(\omega)$ is the harmonic excitation function of the frequency. In this manner, the frequency response of the given equation of motion for forced vibration can be considered aa weighted summation of the single degree-offreedom system responses.

$$
[M]\{x \ddot{(t})\}+[B]\{x(t)\}+[K]\{x(t)\}=\{P(\omega)\} e^{i \omega t}
$$

The structure is tested in a free-free configuration since this is a simple boundary condition that can be achieved in the laboratory. The structure is not attached to anywhere in the model. This boundary condition is used commonly in the literature to correlate the finite element results since this is easy to simulate. Free-free conditions are performed by hanging the structure on light elastic chords or placing the structure on elastic springs or mounts in modal testing. The force is applied from the center point as shown in Fig. 6. This simulates the excitation of the plate by the impact hammer in the modal testing. The acceleration of all other points is calculated to compare the results of the finite element with those of modal testing.

Two types of frequency response analysis are available: (a) the modal frequency response analysis and (b) the direct frequency response analysis. The former is preferred owing to its computational efficiency. This approach uses the mode shapes of the mechanical system. The modes from the modal analysis were used in the frequency response calculation. For this purpose, the modes up to $10,000 \mathrm{~Hz}$ were retained in the solution, thereby meeting the requirement that at least the modes twice the frequency of the range of interest $(5000 \mathrm{~Hz}$ for structure-borne vibration) are included in the calculations. In the calculations of this study, MSC Nastran SOL 111 modal frequency response analysis was used. The accelerance response for each node of the gyroid structure was calculated according to Eq. 3 in MSC Nastran as a measure of the vibrational energy radiated from the gyroid structure.

$A_{i}(\omega)=\frac{a_{i}(\omega)}{P(\omega)}$

where $A_{i}(\omega)$ and $a_{i}(\omega)$ are the accelerance and acceleration of the $i$ th node of the FEM, respectively, and $\mathrm{P}(\omega)$ is the excitation force (the excitation at the center node).

The sum of the accelerance of all nodes of the gyroid structure was taken as the overall measure of the vibration radiated from the structure. Therefore, the overall accelerance, $A_{\text {gyroid }}(\omega)$, of the gyroid structure could be expressed as:

$A_{\text {gyroid }}(\omega)=\sum_{i=1}^{N} A_{i}(\omega)$

The overall accelerance function of the gyroid structure was used to assess the vibration characteristics over the frequency range of interest. Moreover, a key performance metric was defined to quantify the overall vibration characteristics of the gyroid structure as a single value. For this purpose, the root sum of squares (RSS) of the overall accelerance function was selected, as indicated in Eq. 5:

$R S S_{\text {gyroid }}=\sqrt{\sum_{i=1}^{5000} A_{\text {gyroid }}\left(\omega_{i}\right)^{2}}$

where $A_{\text {gyroid }}\left(\omega_{i}\right)$ is the value of the overall accelerance of the gyroid structure calculated at the frequency $\omega_{i}$. In this study, the $R S S_{\text {gyroid }}(\omega)$ metric was calculated up to $5000 \mathrm{~Hz}$.

$O V M=R S S_{\text {gyroid }}(\omega) \cdot m_{\text {gyroid }}$

where $m_{\text {gyroid }}$ is the mass of the gyroid structure.

Therefore, to investigate the dynamic characteristics of the gyroid structure, the parametric studies considered the three metrics: (i) the first mode of the structure, (ii) the RSS of the structure $\operatorname{RSS}(\omega)$, and (iii) the OVM.

\subsubsection{Modal analysis of gyroid structure}

Modal analysis was performed on the DG structure to understand its dynamic characteristics. The results demonstrated that only four modes up to $5000 \mathrm{~Hz}$ existed, which were calculated as 1868.0, 2781.5, 3389.8, and 4391.2 Hz. The mode shapes are illustrated in Fig. 7.

\subsubsection{Comparison of modal characteristics: DG and solid structures}

The dynamic characteristics of the DG structure were compared with those of a solid structure to gain more insight into the dynamic performance. As the modes of the structure were dependent on the mass, the thickness of the solid structure was selected such that the masses of the solid and DG structures were the same. In this case, the thickness corresponded to $6.6 \mathrm{~mm}$ for the solid structure. A similar modeling approach was used to model the solid structure using CQUAD4 surface elements. The same material properties as those of the DG structure were used. The modal analysis results indicated that 13 modes up to $5000 \mathrm{~Hz}$ existed for the solid structure $(580.4,851.5$, 1056.4, 1485.3, 1502.1, 2597.4, 2675.9, 2707.8, 2951.1, 3304.5, 4426.1, 4461.4, and 4938.1 Hz). The first eight mode shapes are illustrated in Fig. 8.

\section{Experimental validation}

The FRF of the structure was also determined by modal testing to verify the results from the FEM. The essence of 
Fig. 7 Mode shapes of DG structure: (a) first mode (1868.0 $\mathrm{Hz})$, (b) second mode (2781.5 $\mathrm{Hz})$, (c) third mode $(3389.8 \mathrm{~Hz})$, and (d) fourth mode $(4391.2 \mathrm{~Hz})$ (a)

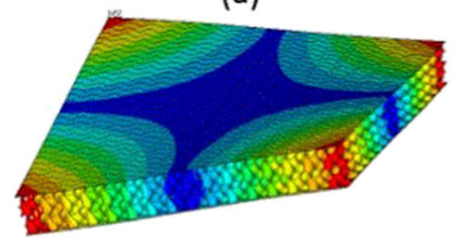

(c)

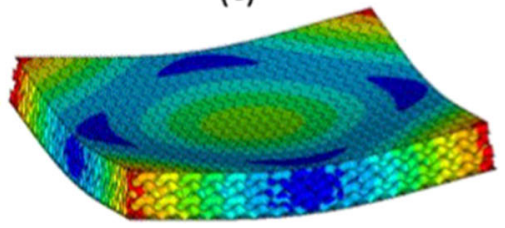

(b)

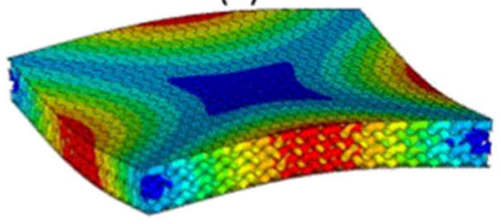

(d)

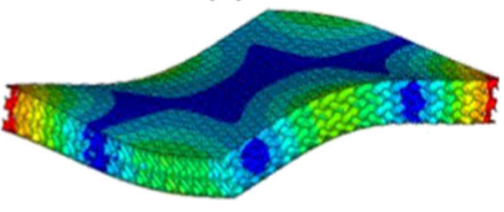

modal testing is measuring the input excitation and system response simultaneously to relate these to one another. The test setup and instrumentation are illustrated in Fig. 9. The mechanical structure was suspended by using flexible cables. Thus, the rigid body modes were substantially lower than the first structural mode of the structure, which was $1868 \mathrm{~Hz}$ according to the modal analysis. The structure was excited at point 13 , as in the simulation, using a DJB IH2 impact hammer. The impact hammer had a force range of $2000 \mathrm{~N}$ and sensitivity of $2.36 \mathrm{mV} / \mathrm{N}$. A hard tip was used to excite the structure in the measurements. During the measurements, the force spectrum was monitored to ensure that the force amplitude did not exceed 10 to $20 \mathrm{~dB}$ at $10,000 \mathrm{~Hz}$, which was the frequency range of interest in this study. The acceleration responses of 24 points (points 1 to 24 in Fig. 9) were acquired using the Dewesoft Sirius data acquisition system. The roving accelerometer method was used in the modal testing. As the structure had high stiffness, the measurement of the response using accelerometers was deemed as sufficient, considering that the mass loading of the accelerometers was minimal. The analyzer provided the data acquisition and signal processing operations. The measurement functions used in the frequency response determination, such as windowing methods and Fourier transforms, were built into the analyzer. Each test was performed three consecutive times and it was ensured that the desired coherence levels were achieved to ensure the accuracy of the modal measurements.

\subsection{Modal analysis}

The results from the modal analysis and modal testing, along with the relative error between these, are summarized in Table 2. Moreover, the mode shapes for the first four modes of the gyroid structure from the FEM and modal testing are compared in Fig. 10. The maximum relative (a)

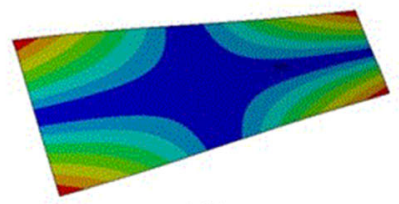

(d)

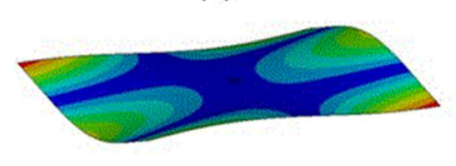

(b)

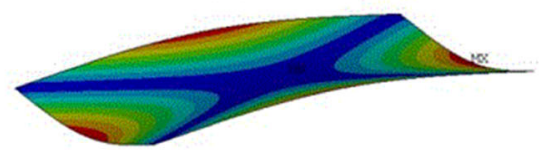

(e)

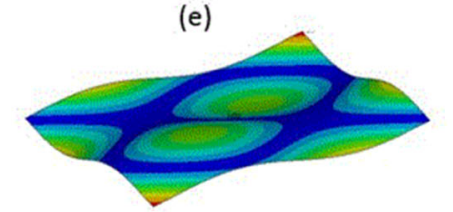

(c)

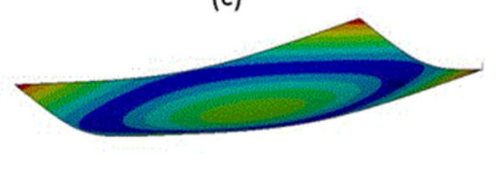

(f)

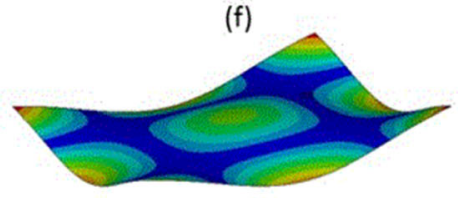

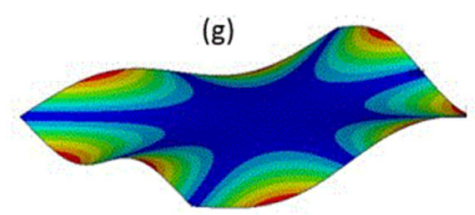

(h)

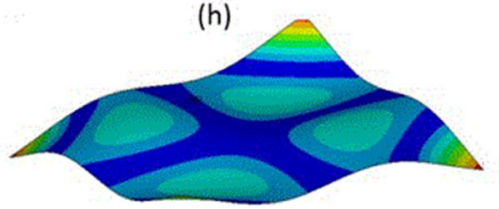

Fig. 8 Mode shapes of solid structure: a mode $1(580.4 \mathrm{~Hz})$, b mode $2(851.5 \mathrm{~Hz}), \mathbf{c}$ mode $3(1056.4 \mathrm{~Hz}), \mathbf{d}$ mode $4(1485.3 \mathrm{~Hz})$, e mode $5(1502.1$ Hz), f mode $6(2597.4 \mathrm{~Hz}), \mathbf{g}$ mode $7(2675.9 \mathrm{~Hz})$, and $\mathbf{h}$ mode $8(2707.8 \mathrm{~Hz})$ 


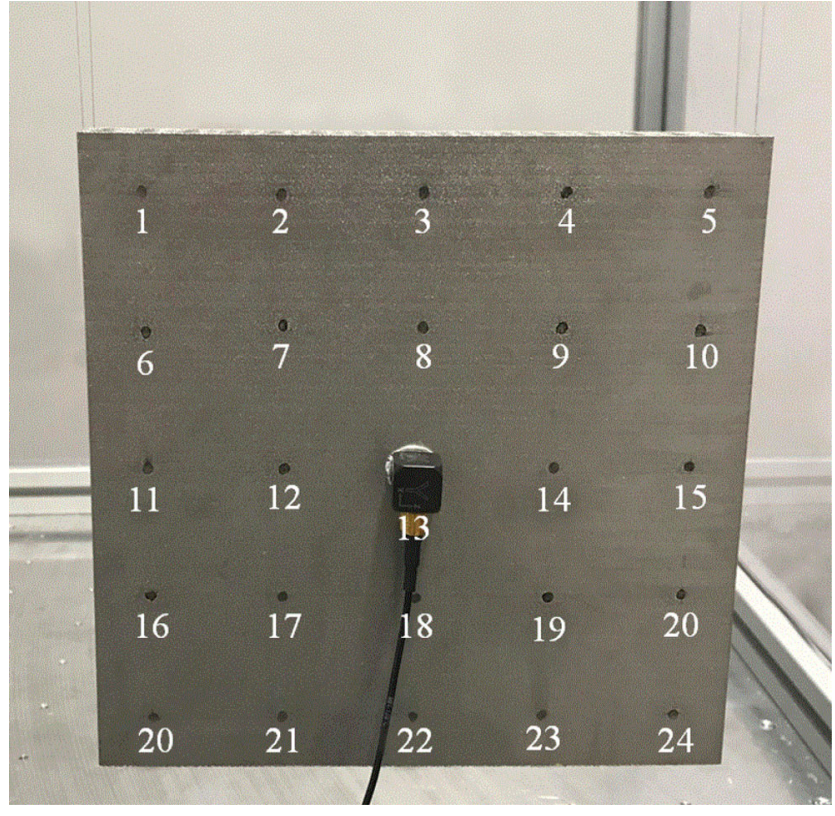

Fig. 9 Modal test setup and hardware

error was $5.5 \%$ corresponding to the second mode. When using the FEM, the first mode could be predicted with $98.8 \%$ accuracy compared with the modal testing. These accuracy levels are quite acceptable for many engineering applications.

\subsection{Analysis of FRFs}

The FRFs from the modal testing and modal analysis were also compared. Only the accelerances of two points were plotted owing to space constraints; however, similar results were obtained for all other points. Results from the modal testing demonstrate that the experimental results were repetitive and the accelerances from the modal analysis fell well within the experimental data. The comparison between the modal testing and Nastran for points 3 and 8 of the solid structure is presented in Fig. 11. Similarly, the

Table 2 Comparison of experimental and simulation results

\begin{tabular}{llll}
\hline Mode no. & Method & Gyroid modes $(\mathrm{Hz})$ & Relative error $(\%)$ \\
\hline 1 & Test & 1890.3 & 1.2 \\
& FEM & 1868.0 & \\
2 & Test & 2636.7 & 5.5 \\
& FEM & 2781.5 & \\
3 & Test & 3221.2 & 5.2 \\
& FEM & 3389.8 & \\
4 & Test & 4447.1 & 1.2 \\
& FEM & 4391.2 & \\
\hline
\end{tabular}

frequency responses from the modal testing and simulation for the DG are illustrated in Fig. 12.

The initial comparison between the FRF from finite element and modal testing is visual. Key metrics for comparison are the location, magnitude, and slope leading to the peaks of the FRFs. The frequencies corresponding to peak FRFs are the modes of the structure. All these key metrics are closely matching between modal testing and FEM results. Since the objective of this paper is to understand the sensitivity of the plate and gyroid wall thickness on the dynamic characteristics, this correlation level is deemed sufficient. The discrepancy between the finite element model and testing may be due to several reasons: First, modeling of the transition region of the gyroid with constant thickness may not be a perfect representation. One remedy could be modeling the structure using $3 \mathrm{D}$ solid elements to represent the transition region more accurately. Second, even though there is no constraint in the finite element model to represent free-free boundary conditions, the modal testing is performed by hanging the structure using the thin elastic cables. The stiffness of the elastic cable still affects the modal characteristics of the structure. Finally, the structure is tested using an impact hammer. While performing the test, it may not be possible to excite the structure at the exact location as the simulation. This partially contributes to the discrepancy of the simulation and experimental results. Another source of error may be attributed to the mass loading of the accelerometers as the mass of the accelerometers was ignored in the FEM model.

Finally, the frequency responses of the solid and gyroid structures for points 3 and 8 are compared in Fig. 13. The visual comparison of the results demonstrates that the number of acceleration peaks of the gyroid structure was less than that of the solid structure. Moreover, the acceleration amplitudes corresponding to the natural frequencies for the gyroid structure were lower than those of the solid structure.

The dynamic characteristics of the solid and the gyroid structures were also compared based on the RSS and OVM metrics. The results indicate that the RSS and OVM metrics of the gyroid structure were lower than those of the solid structure. More specifically, the RSS values of the gyroid and solid structures for point 3 were 3897 and $1975 \mathrm{~m} / \mathrm{s}^{2}$, respectively. Similarly, the RSS values of the gyroid and solid structures for point 8 were 4413 and $2461 \mathrm{~m} / \mathrm{s}^{2}$, respectively.

\section{Parametric studies}

In this section, the dynamic performance of the gyroid structures is investigated by focusing on combinations of 
Fig. 10 Comparison of mode shapes: modal analysis (left) vs. modal testing (right)

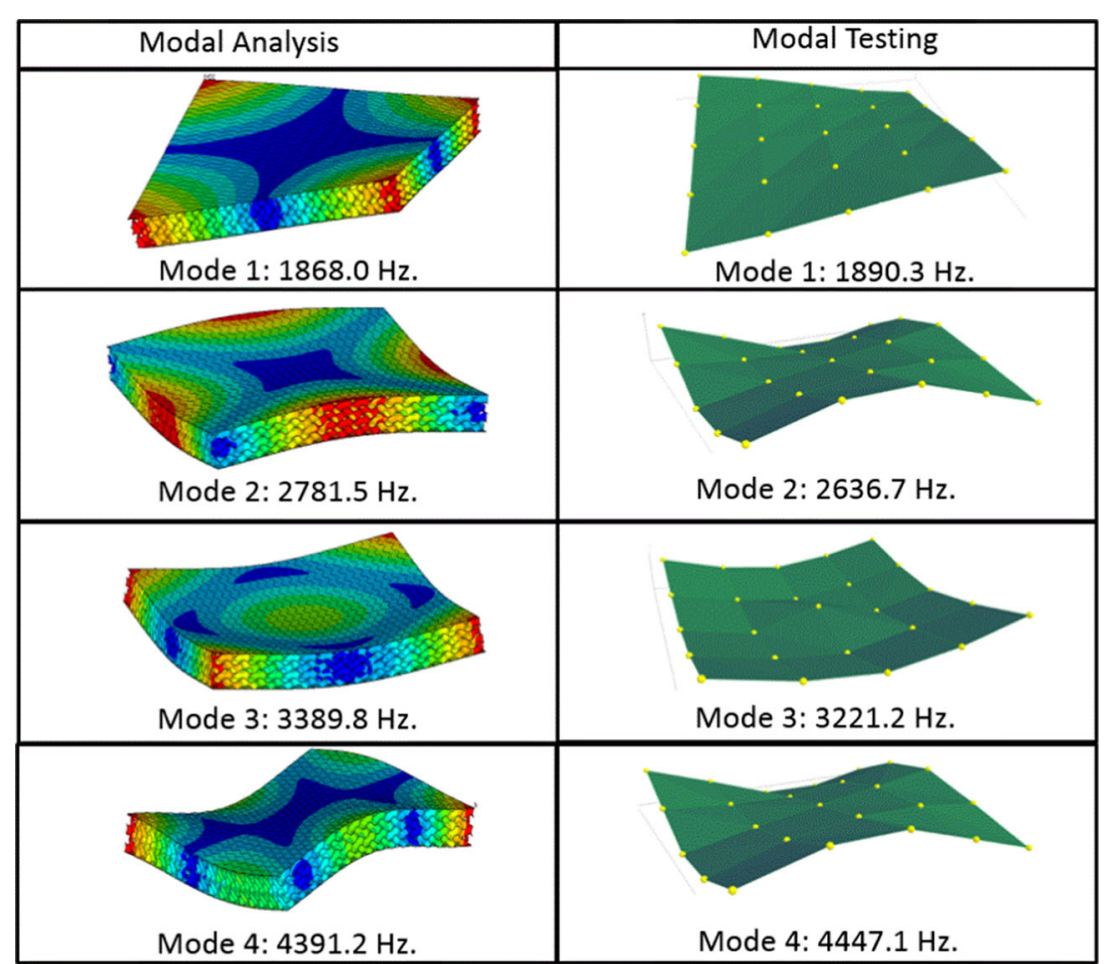

different geometric parameters. To this end, the effects of two design parameters on the dynamic characteristics of the gyroid structure are considered: (i) gyroid wall thickness and (ii) plate thickness. In general, the thicknesses of the plate and gyroid wall improve the overall stiffness of the gyroid structure, and therefore shift the natural frequencies up at the expense of increased weight. Thus, these two design parameters must be carefully engineered to address the trade-off between the modal performance and weight. A parametric study was established to facilitate determination of the aforementioned design parameters. In this study, these

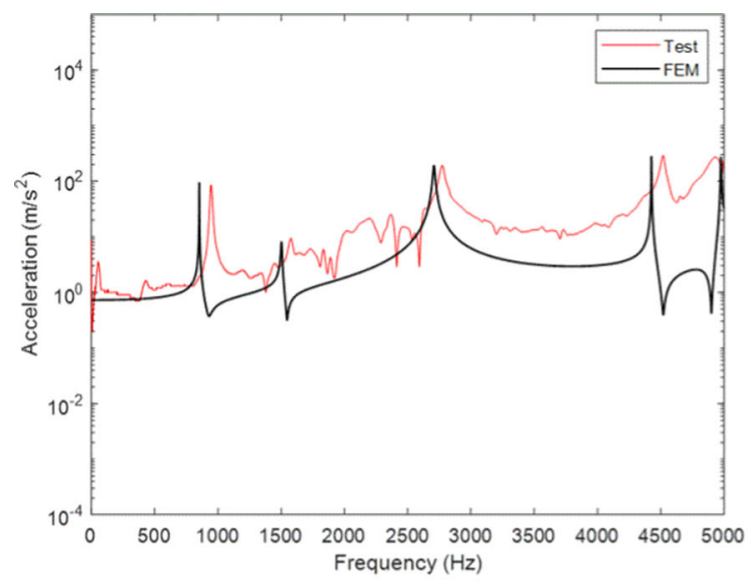

(a) two design parameters were varied across their expected range of limits. In the original design, the gyroid wall and sheet thicknesses were selected as $0.8 \mathrm{~mm}$ without any design consideration. However, for the sensitivity analyses of the design parameters, the manufacturing capabilities were significant. Considering the manufacturing constraints for fabricating gyroid structure configurations with different relative densities, minimum and maximum gyroid shell thicknesses were set to 0.4 and $1.2 \mathrm{~mm}$, respectively. Similarly, the minimum and maximum thickness limits of the upper and lower sheets were selected as 0.4 and $2 \mathrm{~mm}$,

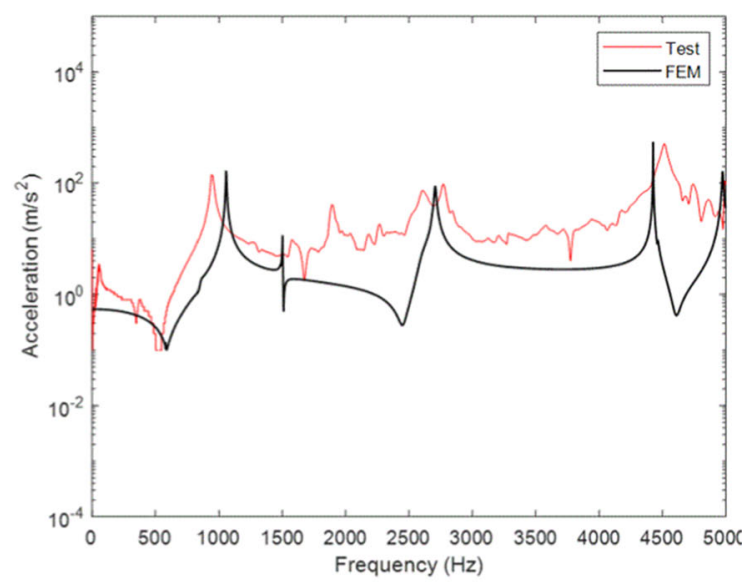

(b)

Fig. 11 Accelerance results for solid structure: a point 3 and $\mathbf{b}$ point 8 


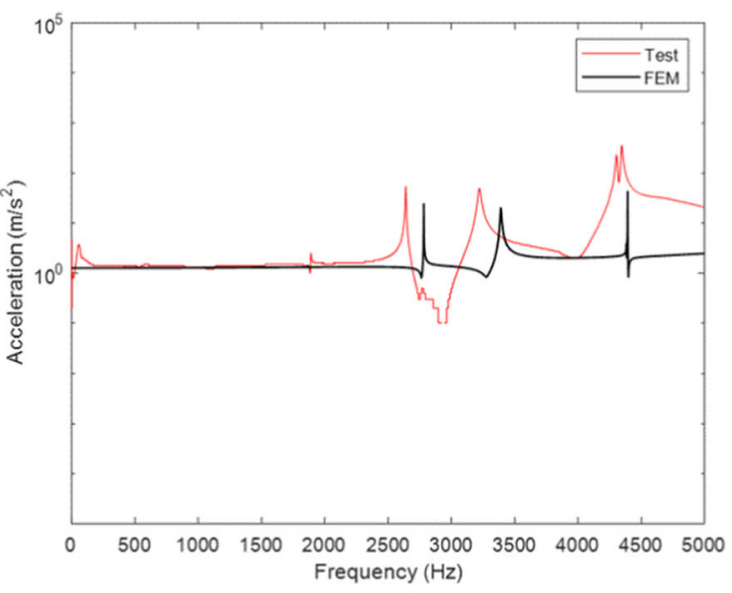

(a)

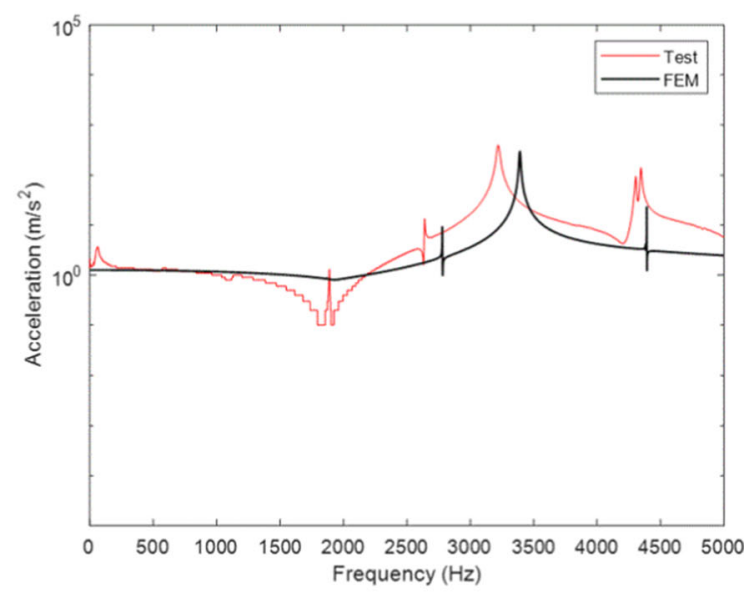

(b)

Fig. 12 Accelerance results for DG structure: $\mathbf{a}$ point 3 and $\mathbf{b}$ point 8

respectively. In the parametric study, the design parameters were incremented by $0.2 \mathrm{~mm}$ for the gyroid wall and sheet thicknesses to generate a homogenous design space. The design configurations are summarized in Table 3. The mass of the design configurations ranged from 1.08 to $3.78 \mathrm{~kg}$. The resolution of the results can be increased using smaller increments on the design parameters for more accurate interpretation of the results if desired.

A script was written to automate the execution and processing of the simulation results, which enabled the simulation of each design configuration without any user interaction. The 45 simulation models were analyzed at the Heavy Computing Center of Ozyegin University using 8 Intel Xeon E5-2680 V4 processors with $8 \mathrm{CPU}$ cores. The CPU time to run the simulation script was approximately $4 \mathrm{~h}$.

\subsection{Effects of design parameters on natural frequencies}

Modal analysis was performed for each design configuration using the methods described in Section 2.3. The results for the first natural frequency with respect to the plate thickness and gyroid wall thickness are presented in Fig. 14. The results of the parametric studies on the second, third, and fourth natural frequencies are illustrated in Figs. 19, 20, and 21 of the Appendix, respectively. It can be observed that the sensitivity of both design parameters in the first natural frequency is significant. In all modes, the curves representing the natural frequencies with respect to the plate thickness at the same shell thickness values tended to be flat, and the effects of the plate thickness on the vibration behavior of the gyroid structures became less pronounced. However,

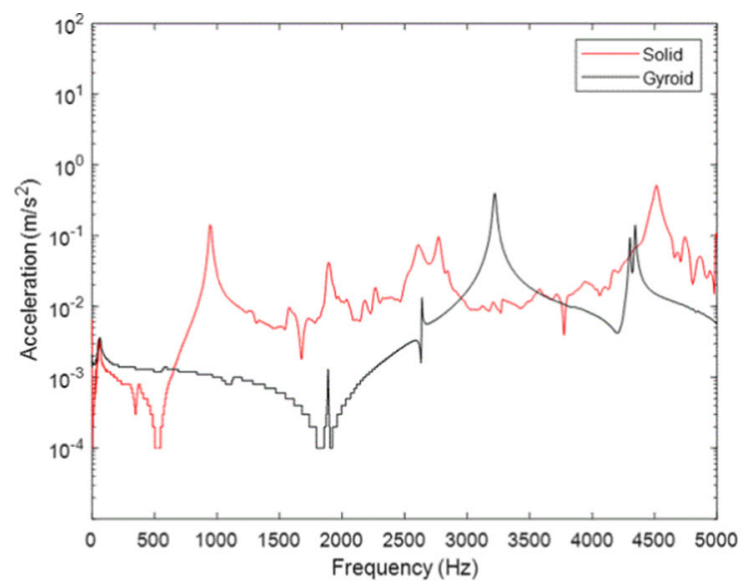

(a)

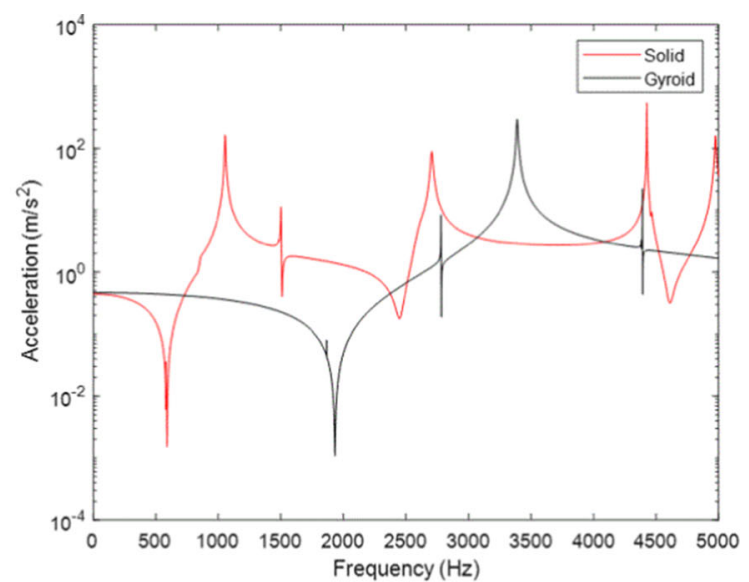

(b)

Fig. 13 Comparison of accelerance results for solid and gyroid structures (point 8): $\mathbf{a}$ test and $\mathbf{b}$ simulation 
Table 3 Comparison of 45 different configurations

\begin{tabular}{|c|c|c|c|}
\hline Design configuration & Plate thickness $(\mathrm{mm})$ & Gyroid wall thickness (mm) & Mass $(\mathrm{kg})$ \\
\hline Configuration 1 & 0.4 & 0.4 & 1.08 \\
\hline Configuration 2 & 0.4 & 0.6 & 1.49 \\
\hline Configuration 3 & 0.4 & 0.8 & 1.90 \\
\hline Configuration 4 & 0.4 & 1 & 2.31 \\
\hline Configuration 5 & 0.4 & 1.2 & 2.72 \\
\hline Configuration 6 & 0.6 & 0.4 & 1.22 \\
\hline Configuration 7 & 0.6 & 0.6 & 1.63 \\
\hline Configuration 8 & 0.6 & 0.8 & 2.03 \\
\hline Configuration 9 & 0.6 & 1 & 2.44 \\
\hline Configuration 10 & 0.6 & 1.2 & 2.85 \\
\hline Configuration 11 & 0.8 & 0.4 & 1.35 \\
\hline Configuration 12 & 0.8 & 0.6 & 1.76 \\
\hline Configuration 13 (original design) & 0.8 & 0.8 & 2.17 \\
\hline Configuration 14 & 0.8 & 1 & 2.58 \\
\hline Configuration 15 & 0.8 & 1.2 & 2.99 \\
\hline Configuration 16 & 1 & 0.4 & 1.48 \\
\hline Configuration 17 & 1 & 0.6 & 1.89 \\
\hline Configuration 18 & 1 & 0.8 & 2.30 \\
\hline Configuration 19 & 1 & 1 & 2.71 \\
\hline Configuration 20 & 1 & 1.2 & 3.12 \\
\hline Configuration 21 & 1.2 & 0.4 & 1.61 \\
\hline Configuration 22 & 1.2 & 0.6 & 2.02 \\
\hline Configuration 23 & 1.2 & 0.8 & 2.43 \\
\hline Configuration 24 & 1.2 & 1 & 2.84 \\
\hline Configuration 25 & 1.2 & 1.2 & 3.25 \\
\hline Configuration 26 & 1.4 & 0.4 & 1.75 \\
\hline Configuration 27 & 1.4 & 0.6 & 2.16 \\
\hline Configuration 28 & 1.4 & 0.8 & 2.56 \\
\hline Configuration 29 & 1.4 & 1 & 2.97 \\
\hline Configuration 30 & 1.4 & 1.2 & 3.38 \\
\hline Configuration 31 & 1.6 & 0.4 & 1.88 \\
\hline Configuration 32 & 1.6 & 0.6 & 2.29 \\
\hline Configuration 33 & 1.6 & 0.8 & 2.70 \\
\hline Configuration 34 & 1.6 & 1 & 3.11 \\
\hline Configuration 35 & 1.6 & 1.2 & 3.52 \\
\hline Configuration 36 & 1.8 & 0.4 & 2.01 \\
\hline Configuration 37 & 1.8 & 0.6 & 2.42 \\
\hline Configuration 38 & 1.8 & 0.8 & 2.83 \\
\hline Configuration 39 & 1.8 & 1 & 3.24 \\
\hline Configuration 40 & 1.8 & 1.2 & 3.65 \\
\hline Configuration 41 & 2 & 0.4 & 2.14 \\
\hline Configuration 42 & 2 & 0.6 & 2.55 \\
\hline Configuration 43 & 2 & 0.8 & 2.96 \\
\hline Configuration 44 & 2 & 1 & 3.37 \\
\hline Configuration 45 & 2 & 1.2 & 3.78 \\
\hline
\end{tabular}




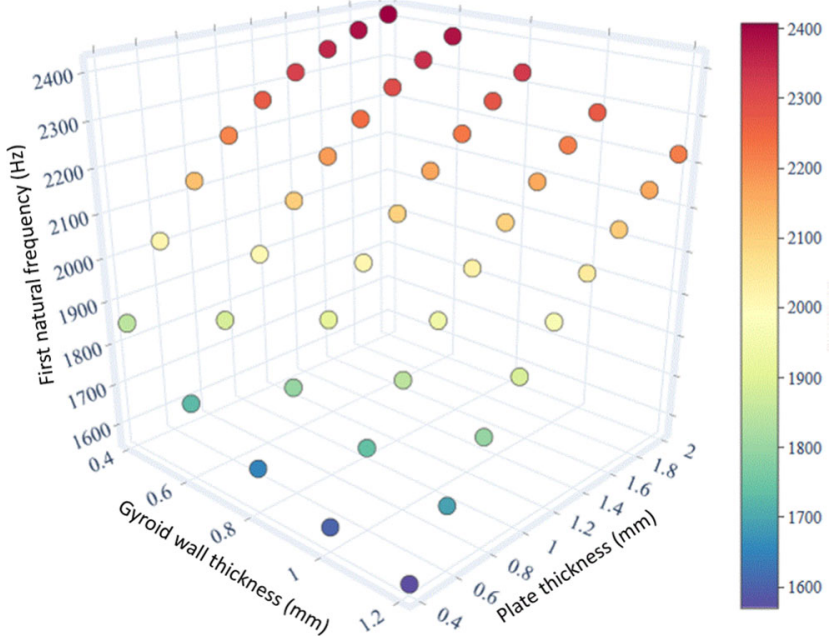

Fig. 14 Design parameters vs. first natural frequency of gyroid structure

a considerable reduction occurred in the first natural frequency of the gyroid structure as the gyroid wall thickness was gradually increased from 0.4 to $1.2 \mathrm{~mm}$. The results of the parametric study also revealed that the highest first natural frequency of the gyroid structure corresponded to the configuration with a plate thickness of $2 \mathrm{~mm}$ and gyroid wall thickness of $0.4 \mathrm{~mm}$. The same conclusions could be drawn for the other natural frequencies.

To address the trade-off between the natural frequency and weight of the gyroid structure, the first natural frequency was normalized by the mass of the gyroid structure, and the results are presented in Fig. 15. The normalized natural frequency results demonstrate that gyroid lattice structures with thinner wall thicknesses provide superior dynamic performance corresponding to the same plate thickness. However, it is not possible to generalize the

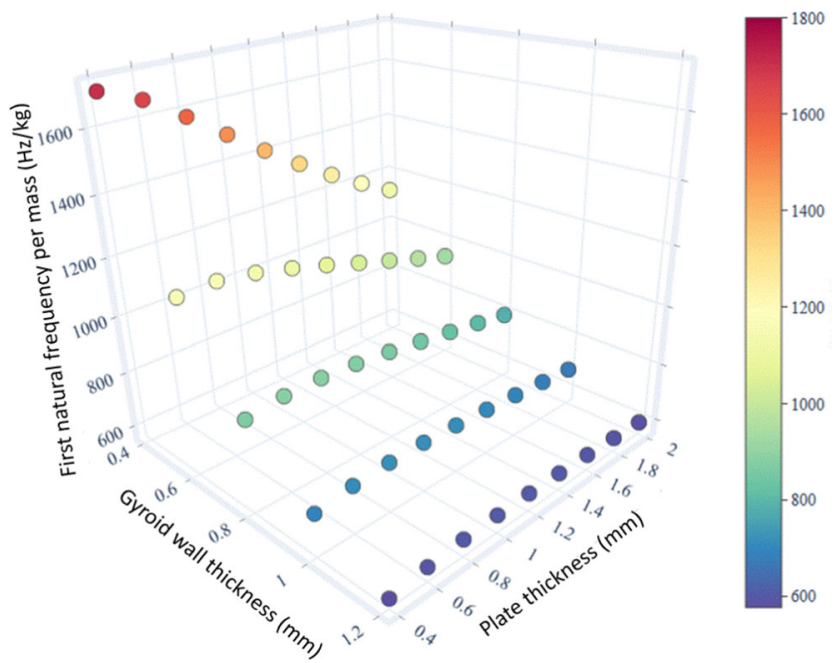

Fig. 15 Design parameters vs. first natural frequency normalized by mass of gyroid structure

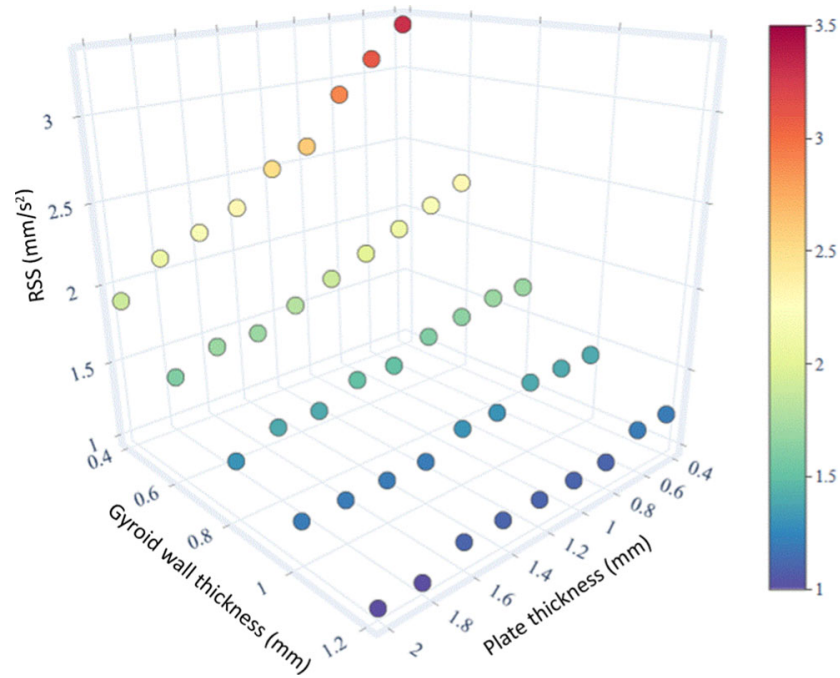

Fig. 16 Design parameters vs. RSS of accelerance of gyroid structure

dynamic behavior of the gyroid structure in terms of changes in the plate thickness at the same gyroid shell thickness. One of the most striking results from the parametric study is that the dynamic performance of the gyroid sandwich structure tended to improve up to the point at which the plate thickness was equal to the gyroid wall thickness. The natural frequency per mass ratio was reduced slightly beyond this ratio. Overall, it can be concluded that the gyroid structure with a plate thickness of $0.4 \mathrm{~mm}$ and gyroid wall thickness of $0.4 \mathrm{~mm}$ exhibited the best dynamic performance among all of the design configurations.

\subsection{Parametric studies on FRFs}

The FRFs for all of the design configurations were calculated, and the RSS of the accelerance from Eq. 5 was determined

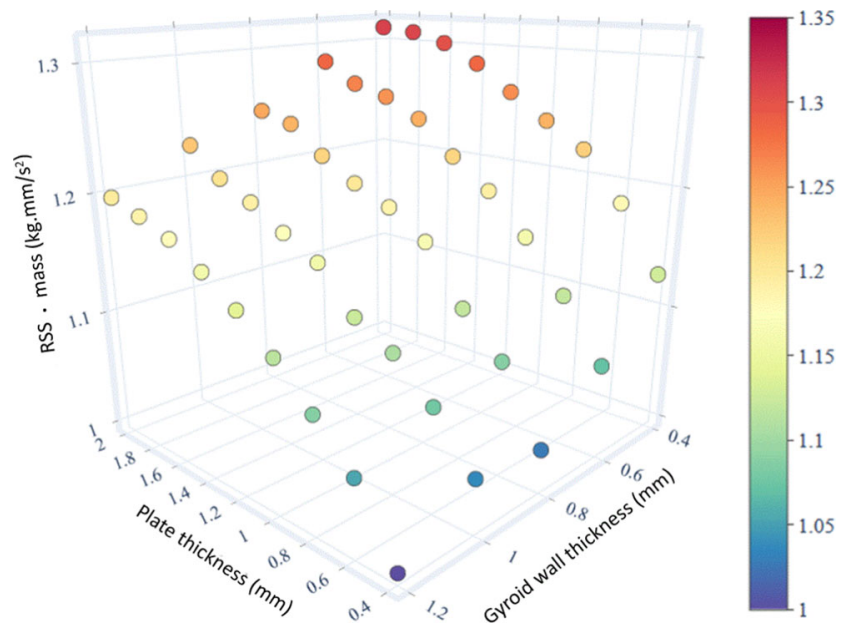

Fig. 17 Design parameters vs. RSS of accelerance multiplied by mass of gyroid structure 
Table 4 Modes of optimized gyroid structure: FEM vs. test

\begin{tabular}{llll}
\hline Mode no. & Method & Mode $(\mathrm{Hz})$ & Relative error $(\%)$ \\
\hline 1 & Test & 1556.5 & 6.6 \\
& FEM & 1666.1 & \\
2 & Test & 2211.3 & 6.7 \\
& FEM & 2370.4 & \\
3 & Test & 2754.7 & 4.0 \\
& FEM & 2869.3 & \\
4 & Test & 3708.9 & 5.0 \\
& FEM & 3903.2 & \\
5 & Test & 3717.9 & 6.4 \\
& FEM & 3970.3 &
\end{tabular}

for each configuration. The RSS of the accelerance of the gyroid structure versus the design parameters is presented in Fig. 16. These results suggest a strong correlation between the RSS and gyroid wall thickness of the gyroid structure. Moreover, the results indicate a nonlinear relationship between the RSS and design parameters. When the gyroid wall thickness was increased, the dynamic responses, defined as RSS responses, dropped off significantly. This trend became more prominent at lower plate thicknesses, such as 0.4 and $0.6 \mathrm{~mm}$. However, no apparent benefits of the dynamic performance of the gyroid lattice structure depending on an increasing plate thickness could be identified with thicker gyroid wall thickness values in these analyses. A positive correlation was observed between the plate thickness and dynamic performance for thinner gyroid wall thicknesses, such as 0.4 and $0.6 \mathrm{~mm}$. Overall, the results of the parametric study suggest that the gyroid structure with a wall thickness of $1.2 \mathrm{~mm}$ and plate thickness of $2 \mathrm{~mm}$ exhibited the best RSS response.

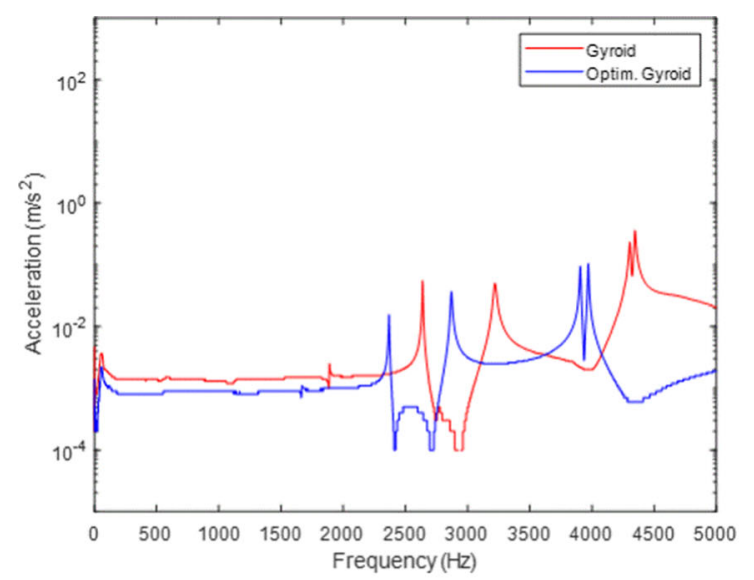

Table 5 Comparison of RSS results from modal testing

\begin{tabular}{lll}
\hline Point no. & Method & RSS $\left(\mathrm{m} / \mathrm{s}^{2}\right)$ \\
\hline 3 & Gyroid & 1975.6 \\
& Optimized gyroid & 492.2 \\
8 & Gyroid & 2461.1 \\
& Optimized gyroid & 1815.2 \\
\hline
\end{tabular}

The proposed parametric study can be used to optimize the significant dimension of the TPMS structures in realworld applications. The parameters can vary across their expected limit ranges as part of a parametric study, and the parameters which yield the desired key performance metrics according to their engineering applications can be chosen in the final design.

Next, the results of RSS.mass were plotted against the design parameters to gain insight into the relationship between the RSS of the accelerance and the mass. Such information was required to make decisions for selecting the design configuration with the minimum mass. The results of the study are presented in Fig. 17. It can be concluded that the metric was reduced as the thicknesses of the plate and gyroid wall were increased.

\section{Verification of parametric design}

The gyroid structure with a gyroid wall thickness of $1.2 \mathrm{~mm}$ and plate thickness of $0.4 \mathrm{~mm}$ delivered the design configuration with the minimum RSS metric. The optimized gyroid structure was manufactured using similar methods to those of the original gyroid structure. The computational weight of the gyroid structure was validated by measuring

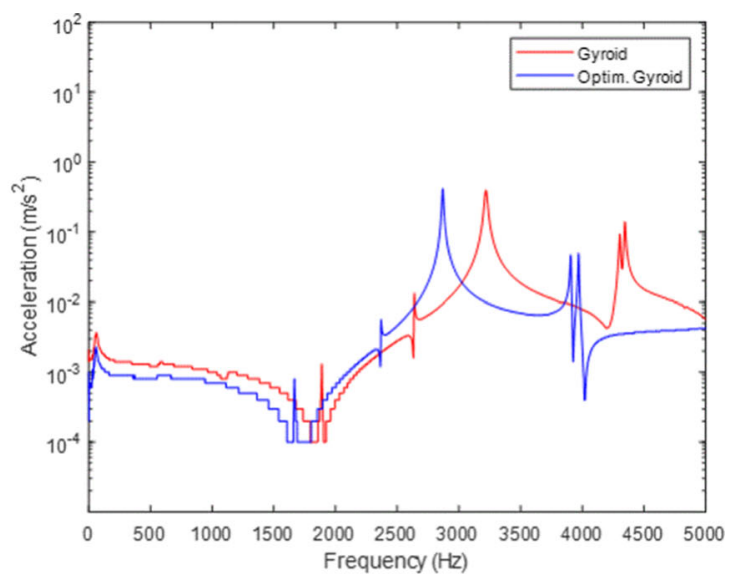

Fig. 18 Frequency response comparison between original and optimized gyroid structures: a point 3 and $\mathbf{b}$ point 8 
a manufactured test sample. The mass difference between the computational model and manufactured test sample was approximately $40 \mathrm{~g}$. The dynamic characteristics of this structure were derived from modal testing. Similarly, modal analysis and frequency response analysis were performed on the FEM of this structure. The modes of the optimized structure from the FEM and modal testing are summarized in Table 4. The results indicate that the relative error between the FEM and modal testing was less than 7\%. The maximum relative error was $6.7 \%$, corresponding to mode 2. Mode 3 was predicted with a relative error of only $4 \%$ between the FEM and modal testing.

The FRFs of original and optimized gyroid structures were compared for points 3 and 8 using the experimental data in Fig. 18. These results were used to calculate the RSS characteristics for the two points indicated in Table 5. The comparison of the RSS for these points demonstrates that the RSS was reduced from 1975.6 to $492.2 \mathrm{~m} / \mathrm{s}^{2}$ for point 3 and from 2461.1 to $1815.2 \mathrm{~m} / \mathrm{s}^{2}$ for point 8 .

\section{Conclusions}

The dynamic performances of gyroid structures made from HS188 were studied and produced by direct metal laser melting using simulation and modal testing. Strong correlation was achieved between the finite element-based simulation model and modal testing. The correlated model was then used as part of a parametric study to understand the effects of the two design parameters on the dynamics of the gyroid structure: (i) plate thickness and (ii) gyroid wall thickness. For this purpose, the design parameters, namely the thicknesses of the plate and gyroid wall, were related to important dynamic metrics, such as the global modes, RSS, and OVM. The design configuration with the smallest RSS value was selected as the optimal design, which was manufactured and verified using modal testing. The simulation results indicated that the gyroidbased sandwich structure exhibits promising attributes for numerous engineering applications in terms of dynamic performance compared with solid geometry with the same mass. However, many other TPMS types exist, such as Primitive, Diamond, and IWP. Therefore, the dynamic performances of the various TPMS topologies should be assessed in terms of their natural frequencies and RSS responses as future work. Although reasonably strong correlation was achieved between the FEM and modal testing, there is room for improvement in the modeling approach. For example, the FEM did not consider the prestress effects associated with the manufacturing process, as the gyroid samples were tested as built, without any heat treatment process. The inclusion of these effects in the FEM will be considered future work. The results demonstrated that simulation and optimization tools can play a significant role in the development of AM, and should therefore form an integral part of AM technologies. Future research may include the application of formal optimization algorithms, such as sequential quadratic programming and genetic algorithms, to determine the optimal design parameters.

Funding This study was carried out under the TUBITAK Technology and Innovation Support Program (Grant number: 5158001).

\section{Appendix}

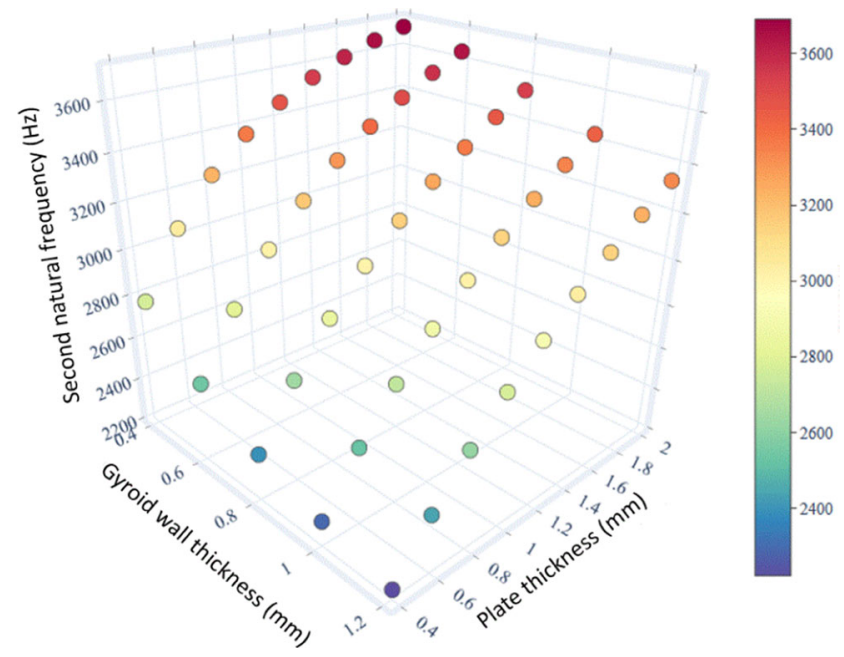

Fig. 19 Design parameters vs. second natural frequency of gyroid structure

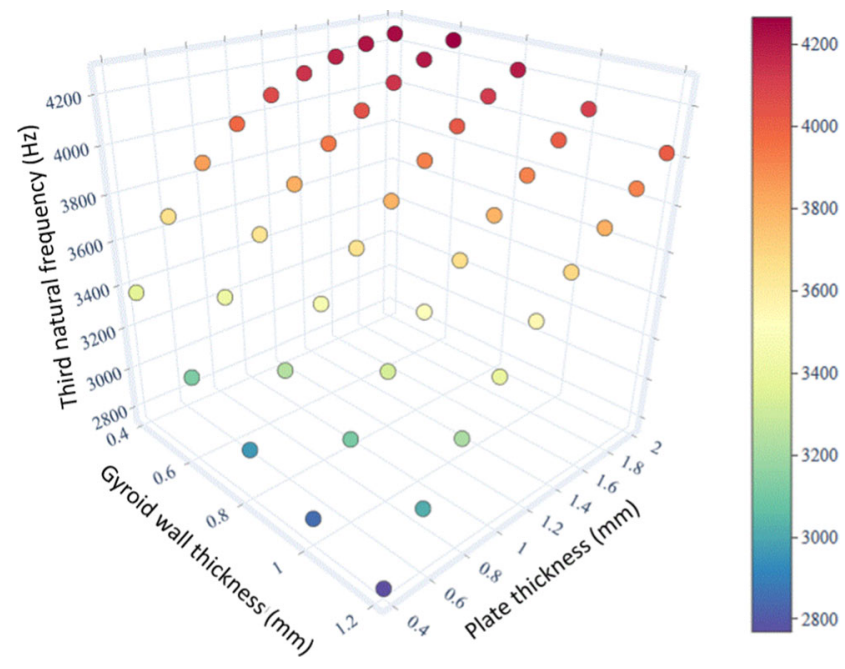

Fig. 20 Design parameters vs. third natural frequency of gyroid structure 


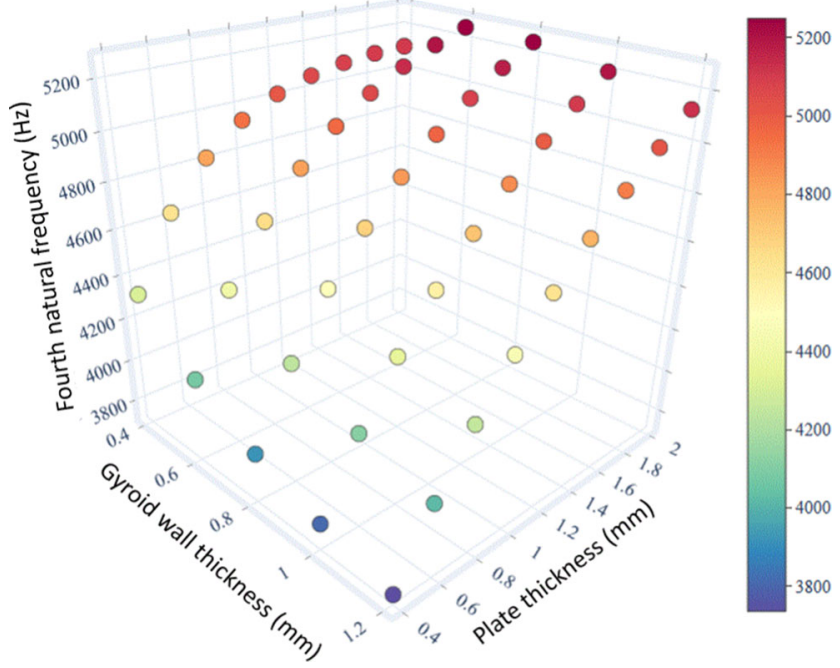

Fig. 21 Design parameters vs. fourth natural frequency of gyroid structure

\section{References}

1. Gibson LJ, Ashby MF (1999) Cellular solids: structure and properties. Cambridge University Press, Cambridge

2. Harrias J, Winter R, McShane GJ (2017) Impact response of additively manufactured metallic hybrid lattice materials. Int $\mathrm{J}$ Impact Eng 104:177-191

3. Ozdemir Z, Tyas A, Goodall R, Askes H (2017) Energy absorption in lattice structures in dynamics: Nonlinear FE simulations. Int $\mathbf{J}$ Impact Eng 102:1-15

4. Kao Y-T, Amin AR, Payne N, Wang J, Tai BL (2018) Lowvelocity impact response of 3d-printed lattice structure with foam reinforcement. Compos Struct 192:93-100

5. Chen L, Zhang J, Du B, Zhou H, Liu H, Guo Y, Li W, Fang D (2018) Dynamic crushing behavior and energy absorption of graded lattice cylindrical structure under axial impact load. ThinWalled Struct 127:333-343

6. Beharic A, Egui RR, Yang L (2018) Drop-weight impact characteristics of additively manufactured sandwich structures with different cellular designs. Mater Des 145:122-134

7. Ferro CG, Varetti S, De Pasquale G, Maggiore P (2018) Lattice structured impact absorber with embedded anti-icing system for aircraft wings fabricated with additive SLM process. Mater Today Commun 15:185-189

8. Wen T, Tian J, Lu T, Queheillalt D, Wadley H (2006) Forced convection in metallic honeycomb structures. Int $\mathrm{J}$ Heat Mass Transfer 49(19-20):3313-3324

9. Wadley H, Queheillalt DT (2007) Thermal applications of cellular lattice structures. In: Materials science forum, vol 539, pp 242 247. Trans Tech Publ

10. Herrmann AS, Zahlen PC, Zuardy I (2005) Sandwich structures technology in commercial aviation. In: Sandwich structures 7: advancing with sandwich structures and materials. Springer, pp 13-26

11. Rupani SV, Jani S, Acharya D (2017) Design, modelling and manufacturing aspects of honeycomb sandwich structures: a review. Int J Sci Eng Dev Res 2:526-532

12. Choy SY, Sun C-N, Leong KF, Wei J (2017) Compressive properties of functionally graded lattice structures manufactured by selective laser melting. Mater Des 131:112-120
13. Kooistra GW, Deshpande V, Wadley H (2004) Compressive behavior of age hardenable tetrahedral lattice truss structures made from aluminium. Acta Mater 52(14):4229-4237

14. Ushijima K, Cantwell W, Mines R, Tsopanos S, Smith M (2011) An investigation into the compressive properties of stainless steel micro-lattice structures. J Sandw Struct Mater 13(3):303-329

15. O'Masta M, Dong L, St-Pierre L, Wadley H, Deshpande V (2017) The fracture toughness of octet-truss lattices. J Mech Phys Solids 98:271-289

16. Tang Y, Kurtz A, Zhao YF (2015) Bidirectional evolutionary structural optimization (beso) based design method for lattice structure to be fabricated by additive manufacturing. Comput Aided Des 69:91-101

17. Wang Y, Zhang L, Daynes S, Zhang H, Feih S, Wang MY (2018) Design of graded lattice structure with optimized mesostructures for additive manufacturing. Mater Des 142:114-123

18. Terriault P, Brailovski V (2018) Modeling and simulation of large, conformal, porosity-graded and lightweight lattice structures made by additive manufacturing. Finite Elem Anal Des 138:1-11

19. Chougrani L, Pernot J-P, Véron P, Abed S (2017) Lattice structure lightweight triangulation for additive manufacturing. Comput Aided Des 90:95-104

20. Panesar A, Abdi M, Hickman D, Ashcroft I (2018) Strategies for functionally graded lattice structures derived using topology optimisation for additive manufacturing. Addit Manuf 19:81-94

21. Du Y, Li H, Luo Z, Tian Q (2017) Topological design optimization of lattice structures to maximize shear stiffness. Adv Eng Softw 112:211-221

22. Maskery I, Sturm L, Aremu A, Panesar A, Williams C, Tuck C, Wildman R, Ashcroft I, Hague RJ (2018) Insights into the mechanical properties of several triply periodic minimal surface lattice structures made by polymer additive manufacturing. Polymer 152:62-71

23. Schoen AH. Infinite periodic minimal surfaces without selfintersections, NASA Technical Reports

24. Yoo D-J (2011) Computer-aided porous scaffold design for tissue engineering using triply periodic minimal surfaces. Int $\mathrm{J}$ Precis Eng Manuf 12(1):61-71

25. Afshar M, Anaraki AP, Montazerian H, Kadkhodapour J (2016) Additive manufacturing and mechanical characterization of graded porosity scaffolds designed based on triply periodic minimal surface architectures. J Mech Behav Biomed Mater 62:481-494

26. Abueidda DW, Bakir M, Al-Rub RKA, Bergström JS, Sobh NA, Jasiuk I (2017) Mechanical properties of 3d printed polymeric cellular materials with triply periodic minimal surface architectures. Mater Des 122:255-267

27. Al-Ketan O, Rowshan R, Al-Rub RKA (2018) Topologymechanical property relationship of $3 \mathrm{~d}$ printed strut, skeletal, and sheet based periodic metallic cellular materials. Addit Manuf 19:167-183

28. Yánez A., Cuadrado A, Martel O, Afonso H, Monopoli D (2018) Gyroid porous titanium structures: a versatile solution to be used as scaffolds in bone defect reconstruction. Mater Des 140:21-29

29. Maskery I, Sturm L, Aremu A, Panesar A, Williams C, Tuck C, Wildman R, Ashcroft I, Hague RJ (2018) Insights into the mechanical properties of several triply periodic minimal surface lattice structures made by polymer additive manufacturing. Polymer 152:62-71

30. Maskery I, Aremu A, Parry L, Wildman R, Tuck C, Ashcroft I (2018) Effective design and simulation of surface-based lattice structures featuring volume fraction and cell type grading. Mater Des 155:220-232

31. Altintas G (2018) Vibration properties of TPMS based structures. Int J Sci Technol Res 4:27-42 
32. Maskery I, Aboulkhair NT, Aremu A, Tuck C, Ashcroft I (2017) Compressive failure modes and energy absorption in additively manufactured double gyroid lattices. Add Manuf 16:24-29

33. Abueidda DW, Elhebeary M, Shiang C-SA, Pang S, Al-Rub RKA, Jasiuk IM (2019) Mechanical properties of 3d printed polymeric gyroid cellular structures: experimental and finite element study. Mater Des 165:107597

34. Elmadih W, Syam WP, Maskery I, Chronopoulos D, Leach R (2019) Mechanical vibration bandgaps in surface-based lattices. Addit Manuf 25(April 2018):421-429

35. Monkova K, Monka P, Zetkova I, Hanzl P, Mandulak D (2017) Three approaches to the gyroid structure modelling as a base of lightweight component produced by additive technology. In: DEStech transactions on computer science and engineering, pp 124-129
36. Kavas B (2019) Compressive strength and characterization of additively manufactured double gyroid lattice structures, Master's thesis, Istanbul Technical University

37. H. International, Haynes 188 properties. https://www.haynesintl. $\mathrm{com} /$, date retrieved 2019-04-20

38. Matweb, Haynes 188 alloy, $0 \%$ cold reduction, $3.2 \mathrm{~mm}$ thick sheet. https://www.matweb.com/search/DataSheet.aspx?MatGUID=868 1846d59904d1086c e346298a79347, date retrieved 2019-04-20

39. Simsek U, Gayir CE, Kavas B, Sendur P (2019) Computational and experimental investigation of vibration characteristic of variable unit cell gyroid structures, Pavia, SIM-AM, 2019

Publisher's note Springer Nature remains neutral with regard to jurisdictional claims in published maps and institutional affiliations. 